\title{
New Developments in the Application of Synchrotron Radiation to Material Science
}

Sunil K. SINHA

Advanced Photon Source, Argonne National Laboratory

Argonne, IL 60439, USA

Recent developments in the application of synchrotron radiation to materials science are discussed, using techniques which exploit the high brilliance of the newer synchrotron sources, such as microbeam techniques and correlation spectroscopy. These include studies of environmental systems, residual stress, slow dynamics of condensed matter systems and studies of liquid surfaces and thin magnetic films.

KEYWORDS: microfocusing, microbeams, microdiffraction, X-ray microscopy, residual stress, coherence, photon correlation spectroscopy, polymer films, magnetic roughness, CMR materials

The last few years have seen rapid advances in the frontiers of materials research, spurred mainly (but not exclusively) by the availability of higher brilliance from the third generation synchrotron radiation (SR) sources (ESRF, APS and SPring-8). While it will obviously not be possible to do justice to all the developments, we shall attempt here to review at least some of the areas which have developed significant scientific momentum. It is probably fair to say that, as expected, the availability of a powerful new enabling tool, namely synchrotron radiation, is leading to real insights into the materials and processes of interest to technology as well as expanding our frontiers in the basic understanding of materials.

One of the most rapidly expanding areas in SR research involves the use of microfocussed X-ray beams." Thus, using devices such as focussing mirrors, multiple refractive lenses, Fresnel zone plates, capillaries, Bragg-Fresnel lenses, and X-ray waveguides, it is possible to focus hard Xray beams down to dimensions as small as $0.15 \mu \mathrm{m}$ (for soft $\mathrm{X}$-rays this can be pushed down to $50 \mathrm{~nm}$ ) with photon intensities of as much as $10^{10}$ photons $/ \mathrm{sec}$. This corresponds to putting into these microbeams orders of magnitude greater photon intensities than were available previously (e.g. from conventional X-ray sources) over macroscopic beam areas. This enables one to do scattering or spectroscopy experiments from micro-regions of the sample and hence enables one to map out structural and other properties of samples on a microscale. This has led to various kinds of imaging microscopies (e.g. phase contrast microscopy, microfluorescence imaging of elemental compositions in samples, micro-EXAFS or near edge spectroscopy imaging of chemical states of atoms in a sample and of course microdiffraction analysis). This in addition to the well-known techniques of $\mathrm{X}$-ray microscopy, and transmission $\mathrm{X}$-ray microtomography and $\mathrm{X}$-ray holography, which have been used to obtain threedimensional real-space images of objects at micron-type is resolution or better. By Liouville's theorem, such high photon intensities in such small areas could not be achieved except using high brilliance X-ray sources, which is why the new SR sources are enabling a quantum leap in such types of studies. (For instance, the focussing of $\mathrm{X}$-rays using glass capillaries from conventional $\mathrm{X}$-ray sources can produce high photon intensities, but the beams are quite divergent and result in relatively poor resolution for diffraction studies, whereas a Fresnel zone plate at a synchrotron can create a spotsize of $0.1 \mu \mathrm{m}$ with an angular divergence of only $0.8 \mathrm{mrad}$ ).

Microfluorescence imaging is beginning to establish itself as an important tool in environmental science, e.g. for the study of the interaction between plants and metal contaminants in solution. Such information is useful for developing an understanding of the transport processes for toxic heavy metals in the environment. $\mathrm{X}$-ray fluorescence has the advantage of much greater sensitivity and penetrating power than other techniques such as charged particle beam techniques. Fig. 1 shows a phase contrast image of a plant root (plantago lanceolata) in association with the mycorrhizal fungal (glomus moseae) with which it has a symbiotic relationship, enabling it to more efficiently extract nutrients from the soil. The spatial distributions of $\mathrm{Mn}, \mathrm{Fe}, \mathrm{Zn}$ and $\mathrm{Cu}$ in a wet root fungus sample have been obtained by scanning with a $1 \mu \mathrm{m} \times 3 \mu \mathrm{m}$ zone-plate-focussed beam and integrating the selected $\mathrm{K} \alpha$ fluorescence on Sector 2 of the Advanced Photon Source. ${ }^{2)}$ The Fe seems to be precipitated preferentially on the edge of the root, while the $\mathrm{Zn}$ is concentrated in the fungal hyphae. Similar types of studies have also recently been made to study the distribution of $\mathrm{S}, \mathrm{Co}$ and $\mathrm{Zn}$ in hydrated bacteria, ${ }^{3)}$ and the uptake of Mn by cancerous cells.

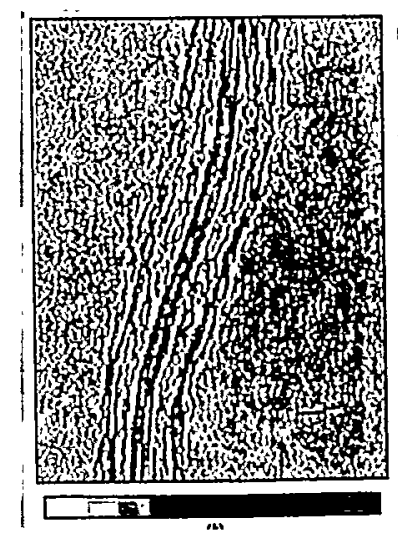

Fig.1. A hard X-ray phase contrast image of an unstained wet $P$. lanceolata root infected by the mycorrhizal fungus $G$. mosscae (from Ref. 2).
The submitted manuscript has been authored by a contractor of the U.S. Government under contract No. W-31-109-ENG-38. Accordingly, the U.S. Government retains a Accordisive royalty-free license to publish or nonexclusive, royaly-iree contribution, or allow others to do so, for U.S. Government purposes. 


\section{DISCLAIMER}

This report was prepared as an account of work sponsored by an agency of the United States Government. Neither the United States Government nor any agency thereof, nor any of their employees, make any warranty, express or implied, or assumes any legal liability or responsibility for the accuracy, completeness, or usefulness of any information, apparatus, product, or process disclosed, or represents that its use would not infringe privately owned rights. Reference herein to any specific commercial product, process, or service by trade name, trademark, manufacturer, or otherwise does not necessarily constitute or imply its endorsement, recommendation, or favoring by the United States Government or any agency thereof. The views and opinions of authors expressed herein do not necessarily state or reflect those of the United States Government or any agency thereof. 


\section{DISCLAIMER}

Portions of this document may be illegible in electronic image products. Images are produced from the best available original document. 
Microdiffraction can be used to study the spatial variation of texture and residual stress across machined or welded structures ${ }^{3)}$. Neutron diffraction has been a highly successful technique used for this purpose ${ }^{6)}$, owing to the great penetrating power of neutrons, but because of brightness limitations, its spatial resolution is limited to $\sim 1 \mathrm{~mm}$. With $x$-rays this can obviously be pushed much further to the extent that the strain can be measured grain by grain in a polycrystalline material (since grain sizes are typically of size $1-\mu \mathrm{m} \sim 20 \mu \mathrm{m}$ ), which leads to the exciting probability of totally describing the stress fields at a truly microscopic level around a propagating crack-tip, for instance. However, the methods for accomplishing this in a routine manner are still very challenging and for penetration into heavy metal components, very high energy $\mathrm{X}$-ray beams ( $>100 \mathrm{KeV}$ ) are required, which are not trivial to focus. For thin film devices, however (e.g. metal interconnects or deposited solid-state quantum well lasers, for instance) microfocussed beams at both the NSLS and the APS are yielding very useful results. Fig. 2 shows the multi-layer diffraction patterns as a function of position (with $1 \mu \mathrm{m}$ resolution) along a multilayer laser deposited on silicon, which is used for optical fiber transmission." The shifts of the multilayer peaks are a measure of the strain as a function of position and show that the "laser" region has a greater tensile strain than the "modulator" region.

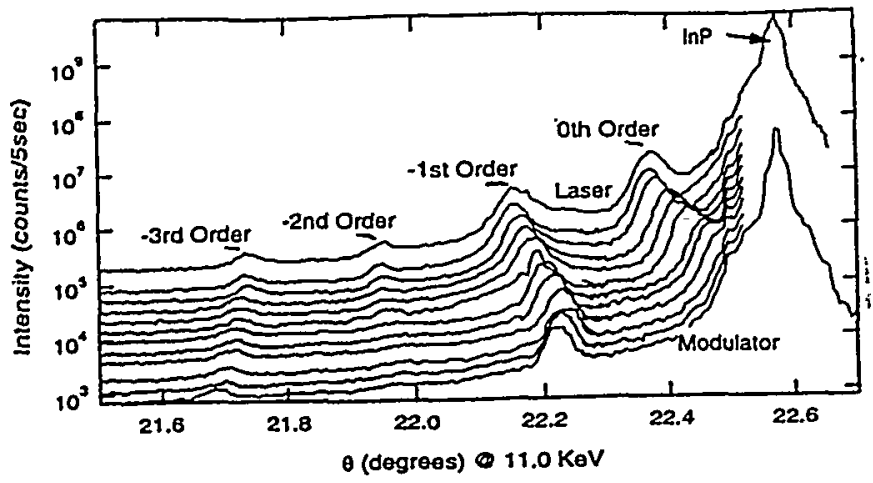

Fig. 2. Bragg scans along the length of a multilayer laser deposited on silicon, approximately $10 \mu \mathrm{m}$ apart (from Ref. 7).

Magnetic circular dichroism (MCD) of circularly polarized $\mathrm{X}$-rays at certain absorption edges of magnetic atoms changes both the real and imaginary parts of the crosssection for the magnetic moment directions parallel or antiparallel to the beam direction. This property can be exploited to map out the magnetic domain structure of a ferromagnetic material, ${ }^{8}$ ) using circularly polarized $X$-rays at the appropriate energy which have been focussed by a zoneplate and scanned across the sample. Other methods of mapping magnetic domains exist of course, much as Magnetic Force Microscopy, but the $x$-ray method is unique in its ability to provide full three-dimensional information (e.g. buried domains).
As a final example of an application of microfocussed beams to study materials problems, we may consider the use of the study of the diffuse scattering from a $0.1 \mu \mathrm{m}$ size microfocussed beam as a function of position from a $\mathrm{Si}$ surface subjected to ion implantation by $1-10 \mathrm{Mev} \mathrm{Si}^{+}$ions (Fig. 3 (a)) carried out by the Oak Ridge group." The diffuse scattering around the $\mathrm{Si}(220)$ reflection was monitored and the line shape was shown to be consistent with interstitial loops at larger depths, yielding a depth distribution which shows that the spatial separation of vacancies and displaced recoiled atoms as expected from calculations. (Fig. 3 (b)).

HIgh Energy, Self-lon Implantation in Si

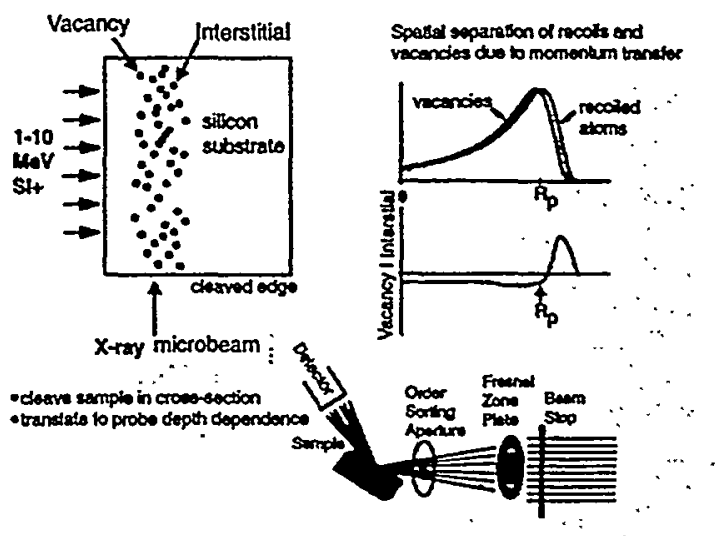

Fig. 3 (a). Schematic of microfocused beam study of self-ion implantation in Si.

\section{Depth-Dependent Diffuse Scattering in Self-Implanted Si}

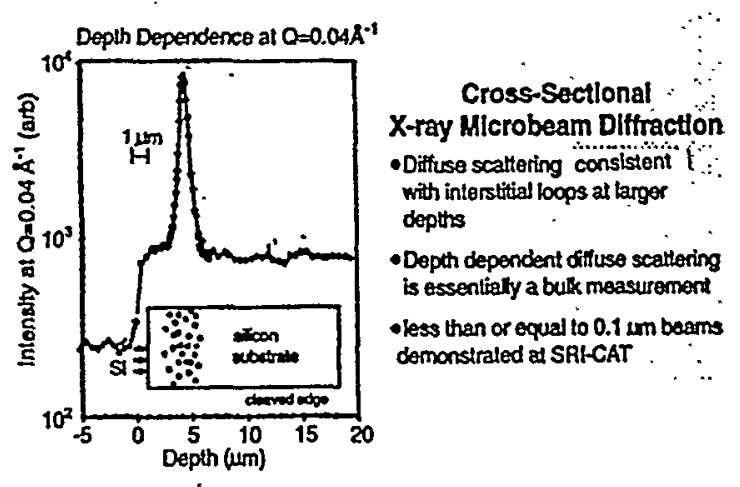

Fig. 3 (b). Depth profile of diffuse scattering intensity from vacancy/interstitial loops (from Ref. 9).

The use of very small-size (though not necessarily microfocussed) beams has another application which is more relevant to basic studies of condensed matter, namely the use of coherent $X$-ray beams to study speckle and the slow dynamics of condensed matter. ${ }^{(0.13)}$ The transverse coherence length of an 
$\mathrm{X}$-ray beam of wavelength $\lambda$ at a distance $\mathrm{D}$ from a source of size $s$ is given by $(\lambda D / s)$, which for sources such as undulator sources at the third generation SR sources translates into distances of roughly $20-30 \mu \mathrm{m}$ in the vertical direction and $\sim 10 \mu \mathrm{m}$ in the horizontal direction. Thus a pinhole of size $\sim 10 \mu \mathrm{m}$ at a distance of $\sim 25 \mathrm{~m}$ from an undulator source will be sufficient to transmit a beam which is entirely coherent in the transverse direction. The longitudinal coherence length is given by $\left(\lambda^{2} / \Delta \lambda\right)$ where $\Delta \lambda$ is the wavelength spread in the incident monochromatized beam. This is typically a micrometer or less, but is effectively magnified by a factor (1/q) for scattering experiments carried out at small wavevector transfer q. ${ }^{(4)}$ The scattering of a coherent X-ray beam from a sample possesses certain unique properties. For instance, any disorder in the sample produces the phenomenon of speckle (well-known in the scattering of laser light) due to the random addition and cancellation of phases of the scattered wavelets from the disordered portions of the sample. It also provides a unique "fingerprint" of the structure of the sample. There are attempts to use this to reconstruct, for instance, the structure of a stepped surface from the speckle pattern of the reflected beam. ${ }^{15}$ ) The timefluctuation of the speckles into and out of a detector from a sample where the scattering units are fluctuating may be used to construct an autocorrelation function for the intensity as a function of time from which one can extract the relaxation time of the dynamics. Specifically, the intensity-intensity autocorrelation function for scattering at wavevector transfer q,

$$
\langle\mathrm{I}(\mathrm{q}, \mathrm{t}) \mathrm{I}(\mathrm{q}, \mathrm{t}+\tau)\rangle /\left\langle\mathrm{I}(\mathrm{q}, \mathrm{t})^{2}\right\rangle=1+\mathrm{g}_{2}(\mathrm{q}, \tau)
$$

where for a system of random particles undergoing diffusion

$$
g_{2}(q, \tau)=C \exp \left(-2 D q^{2} \tau\right)
$$

Here $D$ is the diffusion constant, and $C$ is a prefactor (contrast factor) which is, amongst other things, inversely proportional to the number of "coherence volumes" for the $\mathrm{X}$-rays contained in the illuminated volume of the sample. This is of course the analogue of the well established technique of dynamical light scattering or photon-intensity correlation spectroscopy (PICS) for visible light, but is in principle capable of reaching larger q-values - hence probing shorter length scales in the dynamics. The time scales obtainable with this technique depend on the beam intensity (e.g. on how many photons/sec are scattered into the detector) which is limited for such small beams even at the newest SR sources, but for good scatterers and third generation sources can be as small as milliseconds. Such experiments have been carried out to probe diffusional/relaxational dynamics for colloidal systems 11.12.16) and polymeric systems ${ }^{13)}$. Fig. 4 shows the relaxation time $\left(\left(1 / 2 \mathrm{Dq}^{2}\right)\right.$ plotted vs. $\mathrm{q}$ for a system of block copolymer micelles. Note the deviation from pure diffusional behavior in the form of a peak at the q-value corresponding to a peak in the static structure factor, showing the phenomenon of DeGennes narrowing ${ }^{17}$ (known to neutron scatterers for decades) due to interaction effects, whereby $D$ becomes a q-dependent quantity, inversely proportional to $S(q)$ ) in the simplest theory.

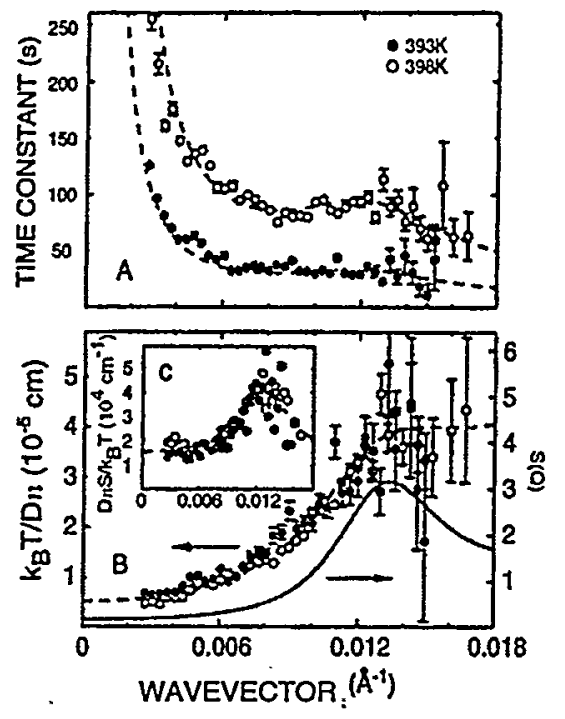

Fig. 4. (A) represents relaxation time for block copolymer

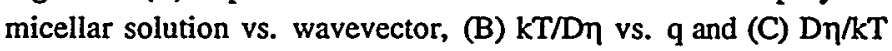
vs. $q$ (from Ref. 13).

Another example of a technique which can be used in the study of slow dynamics, but does not require the use of coherent beams is that of X-ray fluorescent correlation spectroscopy (XFCS) ${ }^{18)}$ In this technique, one measures the autocorrelation of the fluorescence intensity from selected elemental atoms as they move in and out of the beam. The method thus measures the number fluctuations of atoms of this type in the illuminated volume of the sample, and can thus be used to also measure the diffusion and also the sedimentation (or uniform drift) of particles in solution. It has the advantage of being element-specific, since a particular atomic fluorescent emission line can be selected, but does require the use of microfocussed beams and rather dilute systems of large particles (e.g. colloidal metallic particles) since in this case the prefactor of $g_{2}(\tau)$ in Eq. (1) (there is no $q$ involved here) is inversely proportional to the number of particles in the beams at a given time. (An analogue of this method to study rotational dynamics of silicon microparticles in viscous fluids was used several years ago by Wakabayashi ${ }^{19)}$ using an in-house $X$-ray source). Fig. 5 shows the fluorescence autocorrelation function for a series of different sized gold colloids in water from which both the diffusion constants and sedimentation times were extracted and are in excellent agreement with theory. ${ }^{18)}$ Note that the line-shape is very sensitive to the 
form of the dynamics and changes from power-law for diflusion-dominated dynamics to Gaussian for drift-

dominated dynamics. The method can be generalized in special cases to obtain also $\mathrm{q}$ - dependent dynamical information using $\mathrm{X}$-ray standing waves, but this has not as yct been done. Other potential applications include interdiffusion of metals across an interface and (using circularly polarized $\mathrm{X}$-rays) the study of ferromagnetic domain dynamics.

\section{RESULTS: GOLD PARTICLES}

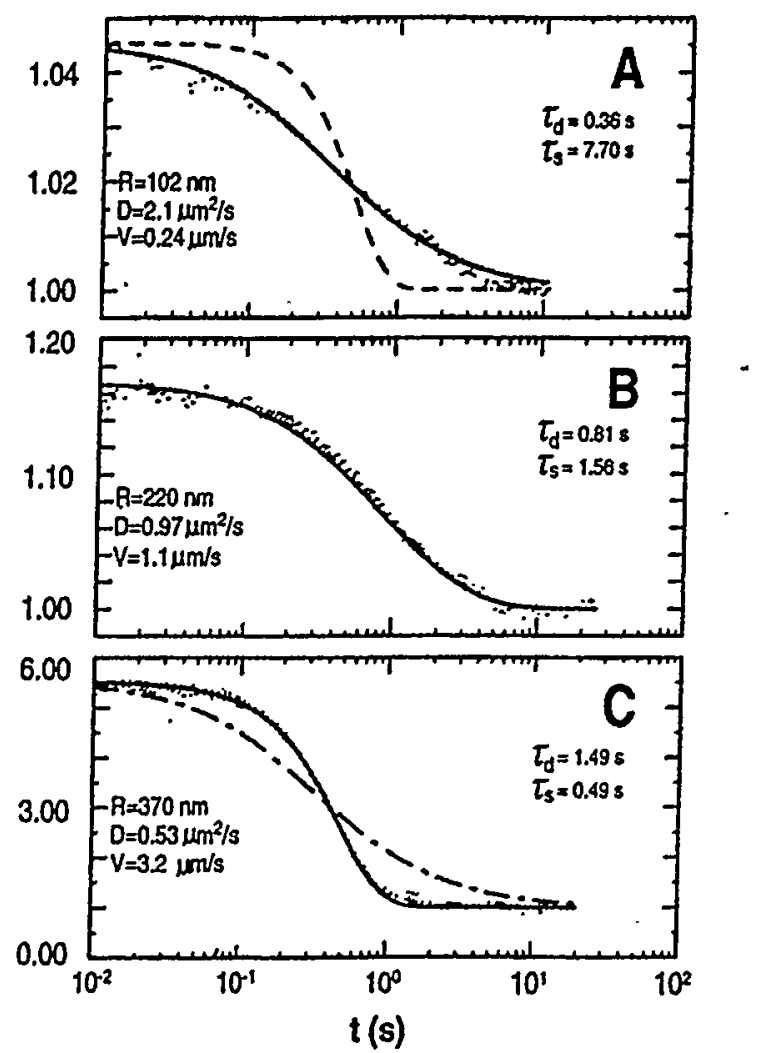

Fig. 5. Fluorescence intensity autocorrelation functions points for solutions of 3 different-sized gold colloids in water. $R, D$ and $V$ represent the colloidal radius, diffusion constant and sedimentation velocity obtained from fitting the curves with a theoretical model (solid curves). The dashed curves in A represents a model with sedimentation only, and in $\mathrm{C}$ a model with diffusion only (from Ref. 18).

Other applications of real-time experiments enabled by high-brilliance $S R$ sources include the study of nonequilibrium phenomena, such as time-dependent EXAFS studies of catalysts under reaction conditions, the growth of domains during phase transformations, and the development of crazing in polymers subjected to stress.

One of the areas where $X$-ray scattering has had the most impact is in the study of surfaces and interfaces. $X$-ray reflectivity and $X$ ray off-specular (diffuse) scattering from surfaces has undergone explosive growth as a popular method for characterizing the roughness, morphology and structure of surfaces, interfaces and thin films.

These techniques often combined with grazing incidence $\mathrm{X}$-ray diffraction (GIXD) have been used to study the structure of multilayers, adsorbed layers, surface reconstruction, oxide growth, corrosion, electrochemically induced surface structural changes, and soft condensed matter systems such as lipid membranes, polymer films, liquid-liquid interfaces, etc. $\mathrm{X}$-ray reflectivity studies of liquid metal surfaces have revealed an intrinsic layering at the liquid/vapor interface as can be seen from the peak at finite $q$ in the reflectivity spectrum from liquid In Fig. 6 (a). ${ }^{20)}$ This was fitted with the oscillating (layered) electron density profile for In (also shown with that for liquid $\mathrm{Ga}$ ) shown in Fig. 6 (b) which is in accordance with the predictions of density functional theory. The effect of capillary wave broadening of the density profile was corrected for in the data. The diffuse scattering from capillary wave fluctuations on liquid surfaces has been studied in detail, ${ }^{20-27}$ and the results are basically in agreement with at least a phenomenological theory based on a free energy expression for the liquid surface with a cut off introduced by a term related to the curvature energy of the surface. The detailed effects of this term have been seen at large in-plane wavevectors as a deviation from the power-law form of the

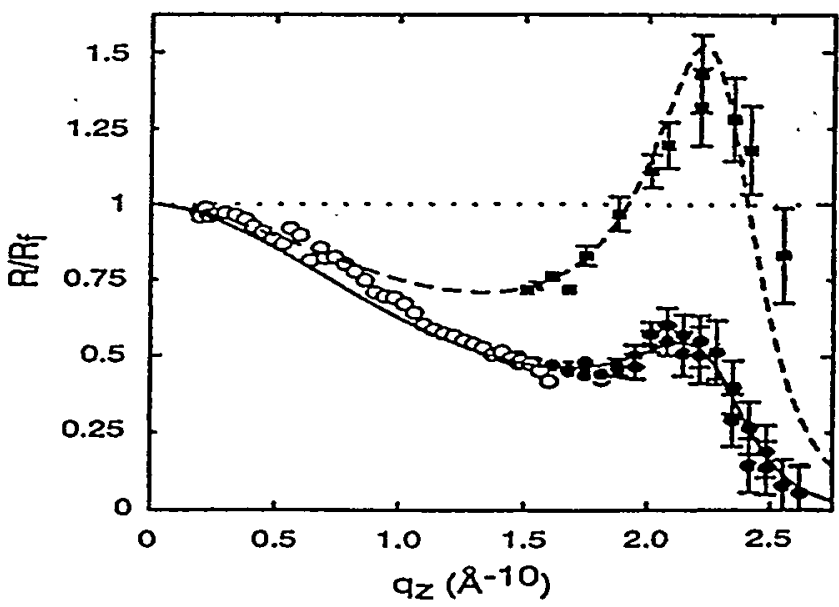

Fig. 6 (a). Specular reflectivity (normalized by the Fresnel reflectivity for a corresponding flat surface) for liquid In at $170^{\circ} \mathrm{C}$ at two different vertical detector resolutions together with model Fits (from Ref. 20). 


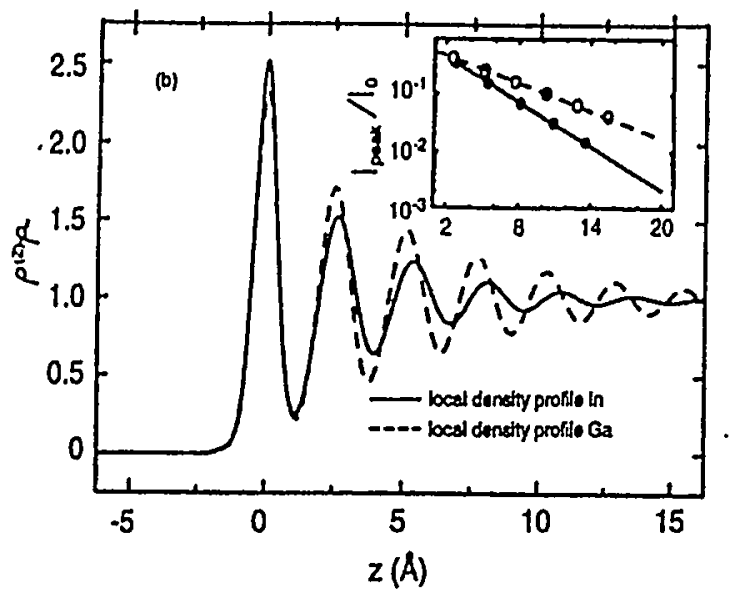

Fig. 6 (b). Real space density profiles for liquid $\mathrm{Ga}$ and liquid In obtained from fitting reflectivity data. Inset represents decay of the amplitudes of the profiles vs. $z$ (Ga: dashed curve; In: solid curve) (from Ref. 20).

scattering $\left(\mathrm{I}(\mathrm{q}) \sim \mathrm{q}^{-(2-\eta)}\right.$ predicted by the (dominant) surface tension term. ${ }^{28)}$ For thin liquid polymer films, the results are more complicated, since the substrate can in principle induce a low wavevector cut-off due to Vander Waals interactions which dominate the much smaller gravitational cutoff wavevector (normally unobservable since it lies well within the instrumental resolution broadening of the specular peak). This causes a flattening of the diffuse scattering profile in a transverse diffuse scan at an in-plane wavevector given by this cutoff wavevector, which in principle should scale as $\left(1 / d^{2}\right), d$ being the film thickness (the effect of the Vander Waals cutoff has also been observed indirectly in neutron reflectivity experiments on polymer films ${ }^{29}$ ). While the above law is obeyed by some polymer films (e.g., poly-vinyl-pyridene films on polystyrene-coated substrates ${ }^{30}$ ) it does not seem to be obeyed for polystyrene films deposited on silicon ${ }^{31)}$ (Fig.

7.) In the latter case, immobile chains on the substrate propagate their effect throughout the film via entanglement, leading to viscoelastic behavior for the film and its surface fluctuations. The effective surface tension of the film is also much larger than that of the bulk polymer liquid, leading to $\eta=0$ in the well-known power-law behavior of the diffuse scattering. Such results are not well understood at present. Capillary wave scattering has also been seen, with its characteristic power-law, from droplets of dewetted polyethylene-propylene (PEP) copolymers on $\mathrm{Si}$ substrates." An interesting casé of capillary wave scattering occurs at the interface of two liquids when their mutual consolute critical point is reached. In this region, the surface tension goes to zero so that the capillary wave fluctuation amplitude becomes very large, and capillary wave scattering is seen even though the contrast between the two phases separated by the interface is going to zero. Such meilsurements have been recently carried out by the MIT group ${ }^{22}$ for the system hexane/perfluorohexane and the expected 3D Ising like exponents were found.

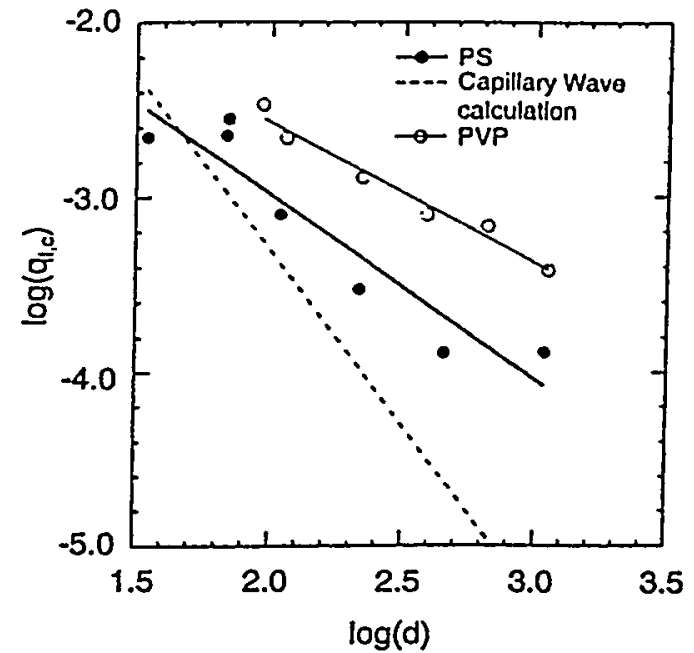

Fig. 7. Low wavevector cut off vs. film thickness for capillary waves propagating on thin liquid polymer films, obtained by fitting the off-specular X-ray scattering from the films. The solid lines are power-law fits of the form $q l c=f / d^{m}$ yielding $m=1.0$ and $\mathrm{m}=0.8$ for PS and PVP, respectively. The dashed line corresponds to the case of free capillary waves with $m=2$ and $b=\AA$ (from Ref. 30).

Off-specular diffuse X-ray scattering studies of ferromagnetic surfaces and thin films have been carried out taking advantage of the large resonant cross section for magnetic scattering at the $\mathrm{L}$ edges of transition and rare-earth metals. By the use of circularly polarized X-rays and measuring both the specular and off-specular scattering for opposite directions of polarization (or of sample magnetization) it is possible to separate the effects of pure charge scattering from that which depends on the magnetization, and hence to separate the roughness and morphology of the chemical and magnetic surfaces. ${ }^{33-35)}$ Such studies are of great importance for thin-film magnetic devices (e.g., magnetic read heads, which incorporate giant magneto-resistive (GMR) films). In general, the magnetic surfaces of the films are found to be smoother than the chemical surfaces, with larger in-plane correlation lengths. In principle, the method could also be used to determine the existence and thickness of unmagnetized "dead" layers at the surface.

As a final example of another basic condensed matter science application of SR, we consider recent experiments on the socalled colossal magneto resistance (CMR) materials embodied in the Lanthanum Manganates. In these materials, the $\mathrm{Mn}$ ions can fluctuate from $(3+)$ to $(4+)$ valence. Double exchange effects favor ferromagnetism at low-temperatures where the delectrons on the $\mathrm{Mn}$ atoms form a metallic state. However, antiferromagnetic and charge-ordered states can also occur, usually accompanied by an insulating phase, and these have been seen with neutron diffraction, electron diffraction and also X-ray scattering. An additional kind of ordering. namely orbital ordering (where the Mn atom d-urbitals arrange themselves in an alternating superstructure) was recently seen in an X-ray experiment on $\mathrm{LaSrMnO}$, by Murakami and co-workers ${ }^{\text {hos }}$ who exploited the 
directional dependence of resonant scattering from such orhitals ("Templeton scattering." "') This is a type of ordering unobservable with other methods. A layered version of these manganite compounds, namely $\mathrm{La}_{12} \mathrm{Sr}_{13} \mathrm{Mn}_{2} \mathrm{O}_{7}$, was recently studied with high-energy (35 $\mathrm{KeV}) \mathrm{X}$-rays where the diffuse scattering was studied around the Bragg points. ${ }^{3{ }^{*} \mathrm{I}}$ An anomalous increase at $\mathrm{Tc}$ (ferromagnetic transition temperature) of the diffuse scattering around the Bragg points was identified with lattice strains induced by an incommensurate charge ordering along the basal plane direction, which manifested itself via a satellite peak at a $\mathrm{q}$ of $(0.3,0,1)$ from the Bragg points. Both the extra diffuse scattering and the charge ordering disappeared discontinuously below $\mathrm{Tc}$ as the charge ordering "melted" in the ferromagnetic phase (see Fig. 8). These experiments showed-r that "polarons" (conduction electrons strongly coupled to the lattice and quasi-localized) do indeed exist in these materials, as has been suggested, ${ }^{39}$ but are more complicated than simple local Jahn-Teller distortions of the oxygen octahedra.
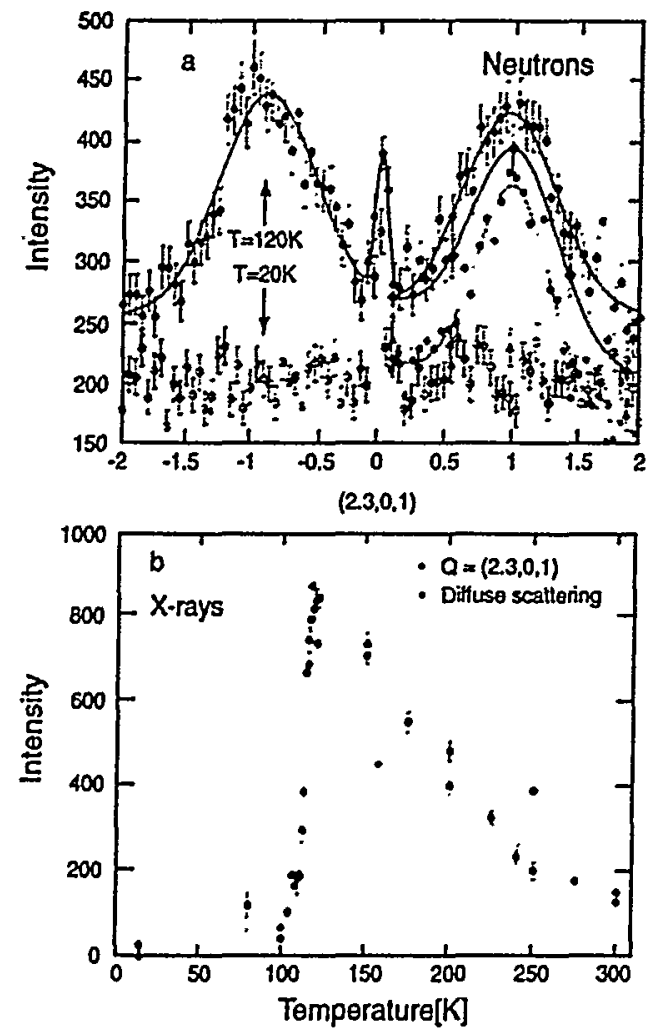

Fig. 8. (a) Peaks in the diffuse scattering at reciprocal lattice points $(2,0, \pm 1)+(0.3,0,0)$ due to charge ordering in $\mathrm{La}_{1.2} \mathrm{Sr}_{1.8} \mathrm{Mn}_{2} \mathrm{O}$, indicating an incommensurate "striped" phase of $\left(\mathrm{Mn}^{3 *}, \mathrm{Mn}^{4 *}\right)$ ordering, but with only short range order..(b) Intensity of the charge-ordering peaks and of the diffuse (Huang) seattering around the Bragg points as a function of temperalure in $\mathrm{La}_{1}, \mathrm{Sr}_{1.8} \mathrm{Mn}_{2} \mathrm{O}_{7}$. The ferromagnetic (mctal-insulator) transition is at approximately $110 \mathrm{~K}$ (from Ref. 38).

While I have picked only a small number of representative recent experiments (obviously the ones I am most familiar with) carried out in the Materials Science/Condensed Matter area, I would like to end by emphasizing that they are only indicative of the vast number of problems being rapidly attacked with the development of new techniques and new instrumentation at SR sources world wide. The recent commissioning of the SPring-8 Synchrotron Source in Japan leads us to anticipate an accelerated impact of such studies on both the science and technologies of the coming decade.

This work has been supported by the U.S. Dept. of Energy Basic Energy Sciences under contract numbers W-31-109-Eng-38 and W-7405-Eng-36. 
1) See "X-ray Microfocusing: Applications and Techniques," (I. McNulty, Editor), SPIE Proceedings, (1998) Vol. 3449 and references therein.

2) W. Yun, S.T. Pratt, R.M. Miller, Z. Cai, D.B. Hunter, A.G. Jarstfer, K.M. Kemner, B. Lai, H.-R. Lee, D.G. Legnini, W. Rodrigues and C.I. Smith, (1998) J. Synchrotron Rad. 5 (1998)

p. 1390.

3) K.M. Kemner, W. Yun, Z. Cai, B. Lai, H.-R. Lee, D.E. Legnini, W. Rodrigues, J. Jastrow, R.M. Miller, S.T. Pratt, M.A. Schneegurt, C.F. Kulpa Jr., and A.J.M. Smucker, Ref. 1, p. 45.

4) B. Lai, et al. Argonne National Laboratory, unpublished information (1998).

5) D. Chidambarrao, Y.C. Song, and I.C. Noyan, "Metallurgical Transactions of Materials A: Physical Metallurgy and Materials Science," (1997) Vol. 28, Chap. 12, p. 2515; T.M. Holden, A.P. Clarke, R.A. Holt, ibid p. 2565.

6) P.C. Brand, H.J. Prask, T. GnaeupelHerold, Physica B, (1997) Vol. 241, p. 1244; X.L. Wang, C.R. Hubbard, S. Spooner, S.A. David, B.H. Rabin and R.L. Williamson, "Materials Science and Engineering A: Structural Materials Properties Microstructure and Processing," (1996) Vol. 211, p. 45.

7) W. Rodriques, Z. Cai, W. Yun, H-R. Lee P. Ilinski, E. Isaacs and J. Grenko: Synchrotron Radiation Instrumentation: $10^{\text {th }}$ U.S. National Conference, ed. E. Fontes, published by AIP Conference Proceeding, Woodbury, New York (1997) p. 161.

8) P. Fischer, G. Schutz, G. Schmahl, P. Guttmann and D. Raasch, Z. Phys. B 101, 3131; G. Srajer, et al. Argonne National Laboratory, unpublished information (1998).

9) B.C. Larson, M. Yoon, J. Tischler and T. Haynes, (to be published).

10) G. Grubel et al., ESRF Newsletter (1994) Vol. 20.

11) S.B. Dierker, R. Pindak, R.M. Fleming, I.K. Robinson, L. Berman, Phys. Rev. Lett., (1994) Vol. 73, p. 82.

12) T. Thum-Albrecht, W. Steffen, A. Patkowski, G. Meier, E.W. Fischer, G. Grubel, D.L. Abernathy, Phys. Rev. Lett. (1996) Vol. 77, p. 5437.

13) S.G.J. Mochrie, A.M. Mayes, A.R. Sandy, M. Sutton, S. Brauer, G.B. Stephenson, D.L. Abernathy, G. Grubel, Phys. Rev. Lett. (1997) Vol. 78, p. 1275.

14) S.K. Sinha, M. Tolan and A. Gibaud, Phys. Rev. B, (1998) Vol. 57, p. 2740; B. Lin, M.L. Schlossman, M. Meron, S.M. Williams, P.J. Viccaro, Rev. Sci. Instrum. (1995) Vol. 66, p. 1.

15) I.K. Robinson, J.A. Pitney, J.L. Libbert and J.A. Vartanyants, Physica B (1998) Vol. 248, p. 387.

16) O.K.C. Tsui and S.G.J. Mochrie, Phys. Rev. E (1998) Vol. 57, p. 2030.

17) Cf. S.W. Lovesey, "Theory of Neutron Scattering from Condensed Matter" (Oxford Univ. Press, Oxford, 1984) Vol. I, p. 247.
18) J. Wang, A. Sood, P. Satyam, Y. Feng, X.-Z. Wu, Z Cai, W. Yun and S.K. Sinha, Phys. Rev. Lett. (1998) Vol. 80, p. 1110.

19) N. Wakabayashi, (unpublished)

20) H. Tostmann, E. Dimasi, P.S. Pershan, B.M. Ocko, O. G. Shpyrko, and M. Deutsch, Phys. Rev. B (1999) Vol. 59, p. 783.

21) A. Braslau, P.S. Pershan, G. Swislow, B.M. Ocko and J. AlsNielsen, Phys. Rev. A (1982) Vol. 38, p. 2457.

22) M.K. Sanyal, S.K. Sinha, K. G. Huang and B.M. Ocko, Phys. Rev. Lett. (1991) Vol. 66, p. 628.

23) J. Daillant and O. Belorgey, J. Chem. Phys. (1992) Vol. 97, p. 5824.

24) E.A.L. Mol, G.C.L. Wong, J.-M. Petit, F. Rieuford, W.H. de Jeu, Phys. Rev. Lett. (1997) Vol. 79, p. 3439.

25) I.M. Tidswell, T.A. Rabedeau, P.S. Pershan, S.D. Kosowsky, Phys. Rev. Lett. (1991) Vol. 66, p. 2108.

26) A.K. Doerr, M. Tolan, T. Seydel and W. Press, Physica B (1998) Vol. 248, p. 263.

27) M. Tolan, O.H. Seeck, J.-P. Schlomka, W. Press, J. Wang, S.K. Sinha, Z. Li, M.H. Rafailovich, J. Sokolov, Phys. Rev. Lett. (1998) Vol. 81, p. 14.

28) C. Fradin, A. Braslau, D. Luzet, M. Alba, C. Courier, J. Daillant, G. Grubel, G. Vignaud, J.F. Legrand, J. Lal, J.M. Petit, F. Rieutford, Physica B (1998) Vol. 248, p. 310.

29) M. Sferrazza, E. Xias, R.A.L. Jones, D.G. Bucknall, J. Webster and J. Penfold, Phys. Rev. Lett., (1997) Vol. 78, p. 3693.

30) M. Tolan, J. Wang, O. Bahr, J. Sokolov, M.N. Rafailovich, S.K. Sinha, Argonne National Laboratory unpublished information (1998).

31) J. Wang, M. Tolan, O.H. Seeck, S.K. Sinha, O. Bahr, M.H. Rafalovich, and J. Sokolov Argonne National Laboratory unpublished information (1998).

32) B.D. McClain, M. Yoon, J.D. Lister, S.G.J. Mochrie, European Physical Journal (1999) in press.

33) J.F. Mackay, C. Teichert, D.E. Savage and M.G. Lagally (1996) Vol. 77, p. 3925.

34) R.M. Osgood, S.K. Sinha, J.W. Freeland, Y.U. Idzerda, S.D. Bader Argonne National Laboratory unpublished information (1998).

35) C.S. Nelson, G. Srajer, J.C. Lang, C.T. Venkataraman, S.K. Sinha, H. Hashizume, N. Ishimatsu, and H. Hosoito, Argonne National Laboratory unpublished information (1998).

36) Y. Murakami, H. Kawaha, H. Kawata, M. Tanaka, T. Arima, Y. Moritoma and Y. Tokura, Phys. Rev. Lett. (1998) Vol. 80, p. 1932.

37) D.H. Templeton and L.K. Templeton, Acta. Crystallog A, (1985) Vol. 41, p. 133; (1985) Vol. 41, p. 365; (1986) Vol. 42 , p. 478.

38) L. Vasiliu-Doloc, S. Rosenkranz, R. Osborn, S.K. Sinha, J.W. Lynn, J. Mesot, O. Seeck, W.-K. Lee and J.F. Mitchell, Argonne National Laboratory unpublished information (1998).

39) A.J. Millis, P.B. Littlewood, and B.I. Shraiman, Phys. Rev. Lett. (1995) Vol. 74, p. 5144. 